\title{
Quaderni
}

QUADERNI Communication, technologies, pouvoir

97 | Automne 2018

Néo-libéralisme(s). Réseaux et formes des

mobilisations en France

\section{La liberté e-limitée ? Structure et générations dans le réseau des militants libertariens français sur internet}

\section{Benjamin Tainturier}

\section{(2) OpenEdition}

Journals

Édition électronique

URL : http://journals.openedition.org/quaderni/1302

DOI : 10.4000/quaderni.1302

ISSN : 2105-2956

Éditeur

Les éditions de la Maison des sciences de l'Homme

Édition imprimée

Date de publication : 5 octobre 2018

Pagination : 69-87

\section{Référence électronique}

Benjamin Tainturier, «La liberté e-limitée ? Structure et générations dans le réseau des militants

libertariens français sur internet », Quaderni [En ligne], 97 | Automne 2018, mis en ligne le 05 octobre 2020, consulté le 04 janvier 2021. URL : http://journals.openedition.org/quaderni/1302 ; DOI : https:// doi.org/10.4000/quaderni.1302 


\section{$D$ ossier}

\section{La liberté e-limitée?}

Aux États-Unis d'Amérique, le mouvement libertarien ${ }^{1}$ a connu un regain d'importance dans le contexte des élections présidentielles de novembre 2016, le candidat libertarien Gary Johnson briguant la présidence. En France, Structure et générations

\section{dans le réseau} des militants libertariens français sur internet ce groupe politique marginal, constitué en un parti pendant l'année $2016^{2}$, s'est simplement contenté de présenter un candidat aux élections législatives de 2017. Cet exemple archétypal accuse la différence de statut qui distingue les idées libertariennes aux États-Unis et en France.

Pour autant, un militantisme libertarien existe bel et bien en France, se fondant sur un répertoire d'actions politiques de plus en plus étendu, certes moins institutionnalisé qu'outre-Atlantique, mais qui profite des technologies numériques et du web ${ }^{3}$. Il permet à des individus libéraux de s'organiser autour d'un contenu militant : réseaux sociaux, blogs, think tanks...

Dans une sociologie du militantisme libertarien français, l'accent mis sur le rôle d'internet est justifié par une raison principale qui a trait à l'histoire croisée des idées et des techniques :

\section{Benjamin Tainturier}

\author{
Diplômé de l'École Normale \\ Supérieure de Paris-Saclay \\ en sociologie et statistiques \\ et de l'ESSEC
} l'internet des origines constituait, pour ses inventeurs, une étape de la réalisation de la grande utopie libérale promue par une certaine contre-culture américaine des années $1960^{4}$. Sébastien Caré, dans Les Libertariens aux États-Unis, définit ainsi l'importance capitale que revêt le web pour les libertariens ${ }^{5}$. Internet ressemble à ce « cyberespace ${ }^{6} »$ modèle de la société du futur', à l'utopie libertarienne réalisée. Partant d'une réglementation minimale au moment de sa création, internet fait émerger un « ordre spontané ${ }^{8} »$ : un ordre non-hiérarchisé, 
auto-organisé, non-centralisé, et surtout nonfinalisé. Des projets en ligne de grande ampleur comme Wikipédia ne cachent ainsi pas leur ascendance libertarienne. Le fondateur, Jimmy Wales, l'avoue et se positionne en bon disciple de Friedrich Hayek, père de la théorie de l'ordre spontané9.

Via le net, il devenait possible d'établir des réseaux de connexions sans devoir imposer à ces réseaux une quelconque centralisation. Cet objectif était très prisé dans la contre-culture libérale. Depuis qu'internet s'est considérablement développé, nous pouvons mettre à l'épreuve une première hypothèse : l'appropriation d'internet par des libéraux et dans un but militant repose-t-elle sur un réseau décentralisé de structures militantes ? Par réseau décentralisé on entend un réseau fait de têtes de pont locales dotées de poids comparables et bien coordonnées, s'exonérant ainsi d'un noyau fédérateur ou d'une clique centrale organisatrice du réseau.

Dès lors qu'est établie l'importance d'internet pour le militantisme libéral, la question des répertoires d'actions des générations militantes se doit d'être posée. Les pratiques numériques des internautes à un instant donné étant fortement déterminées par leur âge, ces pratiques sont très sensibles aux effets de génération ${ }^{10}$. Une étude du rôle d'internet dans le militantisme libéral doit donc mettre à l'épreuve l'hypothèse selon laquelle des générations militantes, aux habitudes militantes différentes, sont identifiables dans le réseau.

Ces deux hypothèses, la première liée à l'aspect décentralisé du réseau de militants, la seconde à l'identification au sein du réseau de générations de structures militantes, nous amènent à poser la question suivante : L'héritage du militantisme libertarien traditionnel français sur la structure adoptée par le réseau des institutions militantes libertariennes françaises sur internet est-il indéniable, ou le dispositif technique qu'est internet redétermine-t-il le militantisme libertarien français en ligne?

Une méthodologie mobilisant à la fois des données massivement aspirées ${ }^{11}$ d'internet et des entretiens avec des militants français rattachés au néolibéralisme sera ici convoquée.

\section{Revue de littérature}

\section{La centralisation du militantisme libéral tradi- tionnel et le clubisme}

La sociologie du mouvement libertarien ${ }^{12}$ nous invite à étudier les think tanks, blogs et sites institutionnels qui fonctionnent sur internet comme des relais d'opinions. Caré démontre en effet que la stratégie de diffusion du libertarianisme vise d'abord à conquérir les esprits, selon une stratégie proprement gramscienne, avant d'envisager l'action directe. L'intellectuel, ce « brocanteur d'idées professionnel ${ }^{13}$ » joue un rôle essentiel. Aux États-Unis, le mouvement libertarien s'est, de fait, cimenté par l'entremise de deux principaux centres de réflexions, prospectifs, et destinés à influencer les pouvoirs publics : le Cato Institute et le Mises Institute. Ces deux instituts contribuent à construire la figure de l'« expert », professionnel à cheval entre les mondes universitaires, politiques, des affaires et 
des médias, et touche-à-tout central dans la stratégie hayekienne de diffusion des idées ${ }^{14}$.

Avant internet, la stratégie militante française est très particulière. Alors que la promotion du savoir libéral aux États-Unis transite essentiellement par les « think tanks » destinés à influencer les politiques publiques, la France ne dispose pas de structure équivalente. Le mode privilégié de la socialisation politique libérale est le clubisme, qui consiste à essaimer, depuis une institution-mère historique, des clubs ou des représentants locaux. Reposant sur un solide maillage territorial, les clubs entretiennent un contact intense avec des publics divers et participent de « la construction d'un sens commun libéral ${ }^{15} »$. La méthode n'est pas circonscrite à une période historique précise : le Club 89 , le Club de l'Horloge, mais aussi l'association Students for Liberty - France, plus récente et à laquelle il est plus simple d'adhérer, constituent des exemples de ce mode d'organisation clubiste. Le clubisme entretient la vitalité des idées libérales, par ses émissaires locaux et le dynamisme d'un noyau central, et confère au mouvement une organisation profondément réticulaire. Les clubs créent du réseau, font se rencontrer et discuter des individus. Sur cette ossature se branchent des compléments qui donnent de l'amplitude à l'appareil principal : les représentants locaux empruntent à des associations plus centralisées et spécifiques des conférenciers et du savoir. Toute l'originalité de la structure du mouvement réside dans ce partage des tâches entre un réseau de personnes qui prennent en charge la sociabilité, et des associations centralisées qui alimentent le réseau en événements, ateliers, conférences. Ces associations dédiées sont nombreuses au $\mathrm{XX}^{\mathrm{e}}$ siècle : le Comité na- tional de la productivité, l'Association française pour l'accroissement de la productivité, le Bureau des temps élémentaires, la Commission générale de l'organisation scientifique du travail.

Le militantisme libéral traditionnel repose donc sur des réseaux fortement centralisés. Ce modèle historique bute contre l'utopie décentralisatrice fondamentale qui explique la popularité d'internet dans les réseaux de la contre-culture américaine des années 1960. Cette opposition entre deux méthodes militantes rend d'autant plus légitime notre premier questionnement : l'appropriation d'internet par des libéraux et dans un but militant repose-t-elle sur un réseau décentralisé de structures militantes?

\section{La variable générationnelle dans le militan- tisme sur internet}

La sociologie des pratiques numériques rencontre ici la sociologie du militantisme. Comprendre comment la variable générationnelle détermine telles ou telles pratiques numériques est un des enjeux de l'édition 2018 de l'enquête sur les pratiques culturelles des Français ${ }^{16}$. Ces données très récentes permettront de confirmer ou d'infirmer une prénotion répandue : l'aisance des plus jeunes publics avec le numérique. Une enquête de 2009 menée par les chercheurs du groupe M@rsouin accrédite notamment cette thèse.

Quelle forme cette appropriation différente d'internet selon les générations peut-elle prendre dans le militantisme en ligne ? On peut déjà imaginer une participation différenciée aux institutions militantes sur internet selon les 
générations d'appartenance des individus et responsables éditoriaux des différentes institutions. C'est ce à quoi nous invite toute la littérature qui cherche à mettre en évidence les déterminants de la participation militante, et qui transpose ces questionnements au militantisme sur internet. A. Théviot ${ }^{17}$ pointe ainsi les effets déterminants de l'âge du diplôme sur le recours à internet comme mode d'expression de convictions militantes. Regrettant dans la plupart des études un manque de considération des " propriétés sociales des militants », Théviot décide de conduire une enquête par questionnaires sur les militants du PS et de l'UMP, ayant des pratiques militantes croisées : certains militent essentiellement dans la rue, d'autres militent en ligne. Elle retrouve ainsi les conclusions de Gibson et Ward dans leur ethnographie du Parti Libéral Démocrate britannique ${ }^{18}$ : les cybermilitants sont plus jeunes. Surtout, le cybermilitant se différencie par un bagage intellectuel plus important, et un diplôme souvent supérieur à la moyenne des autres militants. Pour notre sujet, il faudra donc établir un lien entre génération d'appartenance et position dans le réseau des institutions militantes : les institutions dominées par de jeunes militants sont-elles des points incontournables du réseau militant?

Théviot montre également que les jeunes militants sur internet adoptent des formes de mobilisation plus passives. Elle rejoint ainsi la thèse bien connue de Morozov $^{19}$ : internet et les réseaux socionumériques créeraient une offre partisane d'un nouveau genre, autorisant une forme «fainéante » de protestation. Le militantisme en ligne porterait davantage qu'un réajustement formel de la pratique militante : il modifierait le sens profond du militantisme. Au point de vue empirique, on retrouve les études convaincantes des mobilisations avortées, de ce que M. Lilian ${ }^{20}$ appelle « les mobilisations émergentes ", qui mêlent activistes convaincus, prêts à en découdre, et attitudes consommatrices des soutiens numériques à la grogne. Dans cette perspective, Caroline Frau ${ }^{21}$ développe un argumentaire intéressant touchant à la mobilisation des buralistes en 2007. Elle décrit le rôle de la technique dans les manifestations, dans les regroupements éphémères et dans la diffusion d'anecdotes reconnues scandaleuses. L'auteur interprète l'échec du mouvement comme une preuve évidente de l'inculture militante des nouveaux cyber-militants, et de leur soutien passif, pulsionnel mais rarement porté par des convictions. La prolifération des outils techniques n'a pas aidé au mouvement émergent à se structurer, trop limité par les carences de ses membres, et par le militantisme en dilettante des cyber-activistes. Ainsi, les données qualitatives recueillies pour ce travail permettront de mettre à l'épreuve la prénotion selon laquelle les militants mettent en œuvre un répertoire d'action différent, moins attentif à la doctrine militante, et plus dilettantiste.

Quelle forme l'appropriation différente d'internet selon les générations peut-elle prendre dans le militantisme en ligne? Nous étudierons deux réponses possibles que fournit la littérature : les institutions militantes appartenant à des générations différentes occupent des positions inéquivalentes dans le réseau de la militance, les militants plus jeunes pratiquent un répertoire d'action particulier. 


\section{Données et mesures ${ }^{22}$}

Les données quantitatives utilisées proviennent de divers think tanks, blogs et sites d'organisations influentes dans le mouvement libertarien ${ }^{23}$ en France. Il s'agit d'étudier le réseau qui se tisse entre ces sites en étudiant les pratiques de citations réciproques de ces sites. Plus une institution en cite une autre, plus elle lui accorde de l'importance et la rend visible par les moteurs de recherche sur internet. Les pratiques de citations font donc apparaître les enjeux de réputation qui engagent les institutions militantes sur internet.

Pour établir le corpus des pages web de référence, trois listes de sites ont été enrichies et agrémentées par itérations successives : « http://www. wikiberal.org/wiki/Blogs_lib\%C3\%A9raux », « http://www.wikiberal.org/wiki/Think_tank » et « http://www.wikiberal.org/wiki/Organisations_et_sites_lib\%C3\%A9raux ».

Ces trois sources indiquent des listes de liens utiles à connaître, à visiter et à animer pour les membres de la communauté libérale radicale. Wikibéral présente en effet l'avantage d'être un site à contribution spontanée, en libre accès en lecture comme en écriture, ce qui facilite la mise à jour de la page. Par ailleurs, le caractère libre de Wikibéral lui confère un fonctionnement décentralisé, totalement en affinité avec les convictions libérales radicales des acteurs et des sites que nous souhaitons étudier. Des listes équivalentes existent sur d'autres pages, mais sont toutes le fait d'un auteur en particulier, d'une personne isolée, qu'elle soit administratrice du site, ou auteur influent. Sur Wikibéral, cette liste de liens est le produit d'un travail collectif $^{24}$.

Malheureusement, plusieurs liens indiqués ne fonctionnent plus, sont obsolètes, ou renvoient vers des pages inactives depuis plusieurs années. Un procédé itératif manuel permet de consolider la liste. Pour chaque site présenté sur Wikibéral, les liens externes manifestes mis éventuellement en évidence par ce site, sur les espaces latéraux de la page d'accueil, ou sous cette même page sont ouverts. Pareille mise en scène de liens partenaires, souvent en petit nombre, signifie un lien fort entre les deux pages, une véritable reconnaissance de l'intérêt de l'autre page, pour une cause commune. Sur cette nouvelle page, qui est donc située à distance géodésique de deux liens de Wikibéral, le contenu est étudié pour évaluer sa teneur politique. La présence d'articles mobilisant des auteurs libertariens, (Ayn Rand, Murray Rothbard, Friedrich Hayek ou Ludwig von Mises) est par exemple un bon indicateur de l'affiliation militante des auteurs des sites. Finalement, une fois ce processus itératif terminé, une liste de 71 liens vers des pages d'accueil de sites libéraux radicaux est générée. Par ailleurs, cinq entretiens exploratoires préliminaires avec des militants libertariens ont assuré de meilleurs repères au sein d'une « galaxie » complexe d'idées et d'acteurs, et ont confirmé la qualité des liens présents sur Wikibéral.

À partir du package Rvest de $\mathrm{R}^{25}$, un outil de scraping de liens hypertextes a ensuite été constitué. Le programme circule sur les pages d'accueil de chacun des sites, en extrait les liens hypertextes internes qui renvoient vers une page 
affiliée au site étudié, et les liens externes qui renvoient vers des pages situées ailleurs sur le web. Puis le programme visite une à une toutes les pages internes ancrées dans la page d'accueil, en extrait les liens internes et les liens externes, et recommence par itérations successives, jusqu'à parcourir l'ensemble des pages internes $\mathrm{du}$ site étudié. L'opération reproduite pour chacun des 71 sites de la liste permet de construire une matrice d'adjacence, figurant les réseaux de citations réciproques entre les sites. Cette matrice d'adjacence constitue un matériau commode pour une analyse de réseaux.

Les données qualitatives proviennent de dix entretiens réalisés avec diverses personnalités du militantisme libertarien en France : des professeurs, des responsables d'associations ou de think tanks, et issus de générations diverses.

\section{Résultats}

En considérant toutes les citations qui relient les 71 sites du corpus les uns aux autres, on obtient un réseau très dense et très centralisé. 19 sites en particulier forment entre eux plusieurs cliques de taille 16. Cela signifie que parmi ces 19 sites, il existe de très nombreuses partitions composées de 16 sites qui se citent tous les uns les autres. Les cliques signifient un partage commun de références, et une orientation militante commune. Par ces citations communes, les 19 sites acquièrent une position dominante dans le réseau.

Ces 19 sites sont : Contrepoints, iFRAP, Institut Turgot, H16, Objectif Liberté, Institut Molinari, Extreme Centre, Insolent, NDF, Dreuz. info, Cercle Bastiat, le Blog À Lupus, IREF
Europe, (ces 13 premiers sites sont présents dans toutes les cliques) Institut Coppet, Antagoniste, Fboizard, Économie matin, le blog d'Olivier Demeleunaere, Les 4 vérités. On trouve dans ce groupe les grands représentants de chaque type d'entités, à la fois des think tanks (iFRAP, IREF, Institut Molinari), des journaux (Contrepoints, Nouvelles de France, Économie Matin), des associations (Institut Coppet, Cercle Bastiat), et des blogs (le blog à lupus, H16, Insolent, Olivier Demeleunaere). Également, toutes les tendances du mouvement sont représentées, témoignant de la diversité du militantisme néolibéral, des libertariens et anarcho-capitalistes ${ }^{26}$ (H16, Institut Molinari), des libéraux conservateurs (Nouvelles de France, Insolent ${ }^{27}$ ), des libéraux de gauche sur les questions de société et de droite sur les questions économiques (c'est le positionnement avoué de Extrême-Centre), et enfin des sites aux prises de positions rares (Institut Coppet, Cercle Bastiat).

Si on supprime du réseau les 19 sites identifiés comme les plus centraux du réseau, comment se comportent les 52 sites résiduels? Plusieurs sites forment des cliques de taille 4 : ensembles de quatre sites se citant tous les uns les autres. Cette diminution très importante de la taille de la plus grande clique suggère une véritable organisation oligarchique, dans laquelle 19 sites centraux concentrent l'essentiel des citations. Les sites exclus ne parviennent pas à former de cliques intermédiaires de tailles conséquentes et paraissent ainsi tous très éloignés les uns des autres. Autrement dit, la disparition du réseau originel des 19 sites centraux fait cette fois-ci apparaître un réseau très décentralisé, avec des têtes de pont locales formant des cliques de taille 4 . 
De la même manière qu'on avait fait disparaître du réseau les 19 sites du noyau du réseau, on peut faire disparaître tous les sites qui sont investis dans des cliques de taille 4 dans la version tronquée du réseau. On obtient une troisième version du réseau, dans laquelle aucune clique n'est de taille supérieure à trois. On reproduit le processus jusqu'à n'obtenir que des sites isolés. Il faut pour cela quatre itérations.

Il est alors possible de dégager 5 groupes au sein des 71 sites. On nommera groupe 1 le groupe formé par les 19 sites du réseau de départ investis dans des cliques de taille 16. On nommera groupe 2 le groupe formé par tous les sites investis dans des cliques de taille 4 une fois que l'on a fait disparaître du réseau initial les sites du groupe 1 . On nommera groupe 3 le groupe formé par tous les sites investis dans des cliques de taille 3 une fois que l'on a fait disparaître du réseau initial les sites des groupes 1 et 2 . On nommera groupe 4 le groupe formé par tous les sites investis dans des cliques de taille 2 une fois que l'on a fait disparaître du réseau initial les sites des groupes 1,2 et 3 . On nommera groupe 5 le groupe formé par tous les sites restants.

Partant de ce découpage, on peut réaliser une opération de blockmodelling. Cette opération réorganise le réseau initial en regroupant tous les sites appartenant au groupe 1 dans un même sommet, tous les sites appartenant au groupe 2 dans un autre sommet, tous les sites appartenant au groupe 3 dans un troisième sommet, etc. On conserve tous les liens entre les sommets tels que ces liens existaient dans le réseau des 71 sites et on obtient un réseau de 5 sommets, dont on peut tirer le tableau ci-dessous.

Ce tableau se lit de la façon suivante : Sur 100 liens possibles d'un site du groupe 1 vers n'importe quel autre site, 46 concernent un site du groupe 1, 17 un site du groupe 2. De même, sur 100 liens possibles d'un site du groupe 3 vers n'importe quel autre site, 62 concernent un site du groupe 1, 23 un site du groupe $3 \ldots$

\section{Interprétation : le « bluff technologique » $?^{28}$}

\section{Un réseau très centralisé}

Nous avons vu que le réseau initial était très centralisé. En effet, que 19 sites sur 71 entretiennent des relations si proches est tout à fait remarquable. La disparition de ces 19 sites fait apparaître un réseau à la forme plus décentralisée, où n'existent que des cliques de taille 4, taille faible puisque ce réseau épuré comporte

\begin{tabular}{|l|l|l|l|l|l|}
\hline & \multicolumn{1}{|c|}{$\mathbf{1}$} & \multicolumn{1}{|c|}{$\mathbf{2}$} & \multicolumn{1}{c|}{$\mathbf{3}$} & \multicolumn{1}{c|}{$\mathbf{4}$} & \multicolumn{1}{c|}{$\mathbf{5}$} \\
\hline $\mathbf{1}$ & 0.456959 & 0.1719308 & 0.1705379 & 0.0888426 & 0.1117294 \\
\hline $\mathbf{2}$ & 0.5969166 & 0.22407827 & $\mathbf{0 . 0 6 1 6 8 5 1 8}$ & 0.078459 & 0.03886068 \\
\hline $\mathbf{3}$ & 0.6203753 & $\mathbf{0 . 0 4 9 1 4 7 1 8}$ & 0.23590644 & 0.06552957 & 0.02904151 \\
\hline $\mathbf{4}$ & 0.793157 & 0.14132125 & 0.01092028 & 0.05460139 & 0.00000000 \\
\hline $\mathbf{5}$ & 0.9183191 & 0.05987081 & 0.02181008 & 0.00000000 & 0.00000000 \\
\hline
\end{tabular}


tout de même 52 sommets. En lisant le tableau ci-dessus on trouve que : sur 100 liens possibles d'un site du groupe 1 vers n'importe quel autre site, 46 concernent un site du groupe 1,17 un site du groupe 2, 17 un site du groupe 3, 9 un site du groupe 4 , et 1 un site du groupe 5 ; sur 100 liens possibles d'un site du groupe 2 vers n'importe quel autre site, 60 concernent un site du groupe 1, 22 un site du groupe 2, 6 un site du groupe 3, 8 un site du groupe 4 et 4 un site du groupe 5 .

Inutile de prolonger l'inventaire de ces chiffres pour se convaincre que moins on est intégré avec des petits partenaires locaux, plus on cite des sites canoniques, présents depuis longtemps dans le mouvement, et investissant prématurément les techniques éditoriales propres au web (publications très fréquentes, forte pratique de citations internes, recours aux réseaux sociaux...) : Contrepoints, iFRAP, Institut Turgot, H16, Objectif Liberté, Institut Molinari, Extreme Centre, Insolent, NDF, Dreuz.info, Cercle Bastiat, le Blog À Lupus, IREF Europe.

Contrepoints ${ }^{29}$ est le noyau central du réseau, le site qui rassemble le plus de citations entrantes. Jérôme ${ }^{30}$, un des enquêtés en donne une excellente définition. Se définissant comme libérallibertaire, il récuse la ligne conservatrice qui a pu dominer le militantisme libéral français dans les années $1980^{31}$. Il se définit également comme un militant très actif, créateur d'une structure diffusant des MOOCs sur la pensée libérale et chercheur de trente ans environ. Sur les réseaux sociaux, l'enquêté commente régulièrement les publications de confrères libéraux, et partage lui-même des documents signés de sa main, ou dont il reconnaît l'utilité. Voici com- ment il définit Contrepoints : "Contrepoints c'est vraiment l'exemple typique de ce que sont les libertariens en France: l'auberge espagnole. Il y a des libertariens islamophobes, voilà, très individualistes, il y a des libertariens très conservateurs... le secrétaire de rédaction est conservateur libéral. Le rédacteur en chef est libertarien, pur, en fait dans l'asso ils sont moitié moitié. Ils travaillent tous sous pseudo. C'est la génération au-dessus de moi, l'intermédiaire qui a gardédes liens avec les vieux. Chaque position sur Contrepoints c'est du pour/du contre. Tu vas trouver des articles pour le mariage gay et contre le mariage gay... »

Tous les sites de cet ensemble central de 19 sites, à l'exception de Nouvelles de France, créé en 2011, existent avant 2010 $0^{32}$. Après 2010, tous les nouveaux sites et les nouvelles initiatives libérales qui apparaitront ne pourront donc s'implanter sur le réseau qu'en s'affiliant et se rapprochant des sites dominants de réseaux, qui sont tous antérieurs.

Cette organisation du réseau numérique nous semble donc reproduire une forme de clubisme, forme détaillée ci-dessus et qui est une configuration historique du militantisme libéral en France, où un noyau dynamique et important de militants coordonne une kyrielle d'entités périphériques faiblement liées entre elles. Il n'y a pas eu de succès fulgurant, permis par internet, d'une nouvelle organisation réticulaire décentralisée. Cette technologie n'apporte donc pas cette solution miracle dépeinte par les utopies fondatrices, puisque le leadership qui s'y élabore reprend la configuration du leadership qui y préexistait. 


\section{Position dans le réseau et capital numérique}

Il existe une véritable différence de nature entre les sites du groupe 1, qui forment des cliques de taille 16, et les sites des groupes 2 et 3 . On trouve dans le groupe 2 des sites fameux, comme celui de l'Association pour la Liberté économique et le progrès social ${ }^{33}$, ou des blogs de spécialistes très liés à l'ALEPS, comme Bertrand Lemennicier, Georges Lane, Eric Verhaeghe ${ }^{34}$, et le blog du militant proche de l'extrême droite Claude Reichman. Également, des sites récents comme Écoparis (dédié à populariser l'école autrichienne), TV libertés (site également libéral conservateur avec une ligne identitaire très prononcée), les Éconoclastes, et le blog de Lilianne Held-Khawam forment ce second groupe $^{35}$. Même si ces quatre sites apparaissent après l'année 2010, leurs rédacteurs appartiennent à une ancienne génération libérale, aux « vieux ». Ces entités, dont on rappelle qu'elles citent essentiellement les grandes références du groupe 1, appartiennent à la même génération que les sites du groupe 1, mais réussissent beaucoup moins à s'affirmer sur internet. Elles occupent des positionnements déjà empruntés par

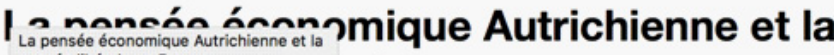 persée libérale en France}

\section{HUMAN

Travail et monnaie, deux valeurs abstraites.

Posted on 13 August 2018

Le mot "valeur" fait partie de ces mots d'économie politique déformés ou dénaturés de
Liens

- ALEPS

- Institut Coppet

- Institut Molinari

- Jörg Guido Hülsmann

Capture d'écran de la page d'accueil du site : https://ecoparis.wordpress.com/ 


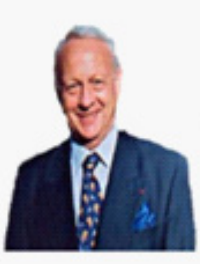

\section{ASSOCIATION POUR LA LIBERTÉ ÉCONOMIQUE ET LE PROGRES SOCIAL}

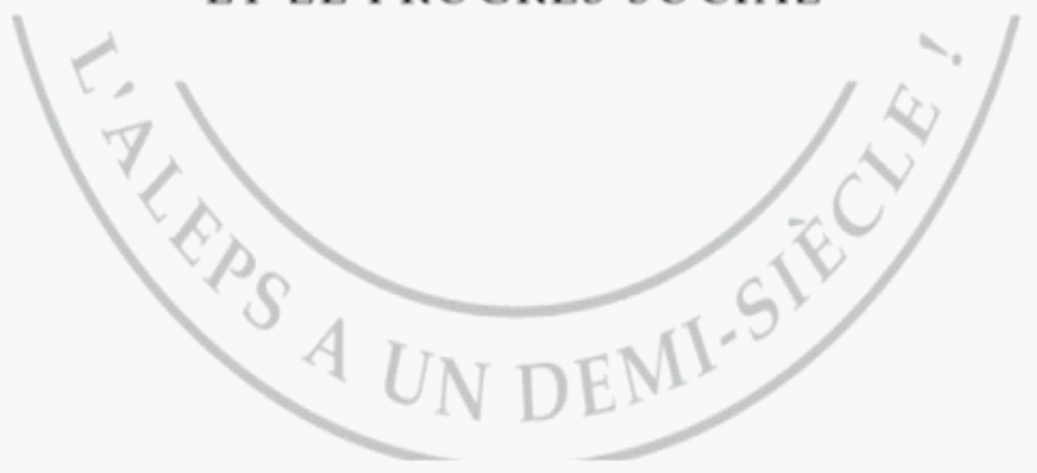

\section{Plan du site}

\section{I0 Actualites}
Actualités prochaines
Din Demière
If Actualités antéricures

Capture d'écran de la page d'accueil du site http://www.aleps.paris/sitemap/ 
des têtes de pont (c'est le cas pour Écoparis, Les Éconoclastes, TV libertés) où suivent des supports moins solubles dans le dispositif technique qu'est internet ${ }^{36}$. Ce groupe assez homogène et sans dynamique interne notable, très tourné vers le noyau du réseau, présente le résidu militant du mouvement, qui n'est pas parvenu à s'implanter en ligne. Voici quelques captures d'écran datant de l'été 2018, gràce auxquelles on se rendra compte d'une certaine vétusté dans l'éditorialisation des différents sites, preuve d'une mauvaise dilution du contenu militant historique dont ces sites sont dépositaires, dans les codes du militantisme sur internet.

L'étude des sites appartenant au groupe 3 révèle une configuration tout à fait différente. On identifie dans ce groupe des sites très récents et tenus par de jeunes militants, qui sont les sites qui donnent une impulsion nouvelle au mouvement : Génération libre, Think libéral, Students for liberty, École de la liberté, Collectif Antigone. On invitera le lecteur à comparer les interfaces ci-dessus aux pages d'accueil très dynamiques et graphiques des sites Génération Libre, Students for Liberty ou Think libéral par exemple.

Pour bien comprendre la position des différents sites dans les réseaux de citations, il y a donc deux critères à prendre en compte. D'abord la réputation du site, exogène au réseau, qui provient de l'intensité de son militantisme, des responsables qui l'animent. À cela s'ajoute la maîtrise des codes du web, des outils de valorisation des articles et des contenus publiés en ligne. Internet, en quelque sorte, génère son propre capital, une forme de « capital numérique » que les acteurs ne possèdent pas uniformément, et qui jauge la maîtrise du dispositif technique en tant que tel. Les sites qui dominent le réseau présentent des avantages dans ces deux domaines : ils bénéficient d'une forte notoriété hors du web et exploitent l'outil numérique au mieux. Composant un second ensemble, on trouve les acteurs historiques du militantisme, qui ont une position plus périphérique dans le réseau en dépit d'une bonne notoriété. Ils pâtissent de leur méconnaissance des outils numériques. Enfin, les jeunes sites, souvent portés par des initiatives étudiantes ou des jeunes militants rompus au technologie du web, mobilisent le web pour renouveler les pratiques militantes. Voilà comment un second enquêté, Franck, me décrit le nouveau répertoire d'actions qu'il travaille à mettre en œuvre. L'homme est âgé de 27 ans et est coordinateur national pour une association libertarienne étudiante internationale. Il est également proche du site Génération Libre, et m'avoue avoir franchi une étape décisive dans sa conversion aux idées libérales radicales après la lecture de Ayn Rand. Il est également passionné par la disruption et le hacking, formes contemporaines d'utilisation de la technique pour débarrasser certains services d'intermédiaires ou de pesanteurs administratives qui brident les usagers desdits services.

«On est en train d'inventer des choses, il y a dix ans, il n'y avait rien. On cherche à éduquer les gens, certains ne savent pas nous positionner. On est plus là pour créer une sorte de climat, favorable au changement! On n'est pas des lobbyistes hein! Il faut que tu montres aux gens comment eux ils ont le pouvoir, comment eux ils peuvent le prendre. Il faut regarder tous les contre-projets qui existent tu vois? Et tu fais 
une espèce de "big picture 》, tu montres ce que c'est que la politique, la vraie. Faut montrer des contre-modèles et montrer que c'est par et pour ces contre-modèles que tu veux moins d'État, moins de politiques coercitives... C'est la radicalité dans le pragmatisme: t'es contre l'état d'urgence, t'as une position radicale làdessus, tu sais que politiquement tu vas pas faire avancer grand chose, bon, en mode think tank tu peux déposer un recours devant le conseil d'État, et pis en attendant tu peux échapper au gouvernement en chiffrant tes mails et tes messages. Là, tout de suite, c'est ça un anarchisme pragmatique. Et t'avances sur ces trucs, et du coup tu fais pas peur à grand monde, et puis tu changes petit à petit le quotidien des gens. »

\section{La mise en évidence d'un clivage intergénéra- tionnel}

Terminons par le point le plus notable de cette étude, notifié par les deux chiffres en gras dans le tableau ci-dessus : sur 100 citations possibles envoyées par le groupe 2 vers d'autres sites, seules 6 concernent un site du groupe 3. Inversement, sur 100 citations possibles provenant du groupe 3, seules cinq sont envoyées en direction de sites du groupe 2. Ces deux chiffres soulignent l'important clivage intergénérationnel qui frappe le réseau. Le groupe des « vieux » refuse de renvoyer aux sites du groupe des « jeunes » et vice-versa. Pourquoi cette communication difficile ? Certainement parce que les répertoires d'actions collectives diffèrent sensiblement si l'on en croit Jérôme, jeune chercheur préalablement cité, qui me confie : "Ils ont un usage des réseaux sociaux qui me dépasse, ils sont impossibles à cadrer ces types! Il y a une dimension communautaire très forte, on boit des bières, on fait des soirées hacking machin truc, et moi je t'avouerais que cela me dépasse complètement. C'est un autre type de militantisme, plus communautaire... Moi je ne suis pas certain que cela paie à l'avenir... il faut en rester aux idées, lire Hayek dans le texte. On a plus l'impression que ce sont des gens qui se complaisent dans leur activité subversive mais cela ne forme pas des militants qui seront là dans vingt ans. »

Le jeune chercheur interrogé me confirme ce point en interview, soulignant l'importance du clivage intergénérationnel repéré ci-dessus : « $A$ l'époque tu arrivais au néo-libéralisme par ces réseaux là, c'était l'ALEPS qui avait le monopole sur le libéralisme, la bibliothèque sur le libéralisme. Et là, on voit, depuis 10 ans, une arrivée de nouveaux... qui ne consultent même pas J. Garello et compagnie, et cela crée une espèce de libéralisme, libertaire, assumé comme tel. Qui n'existait pas il y a dix ans. Moi j'arrive à leur première réunion, il y a dix ans. Une réunion sur l'éducation, je me dis ouais trop bien on va se débarrasser des profs tout ça (parce que moi je suis libéral-libertaire), et là j'arrive ils disaient qu'il fallait promouvoir l'éducation privée, comme ça on allait pouvoir protéger nos enfants de l'influence des gauchistes, les élever chez nous et leur apprendre les valeurs chrétiennes... 》

Avant d'ajouter plus loin que le répertoire d'action collective diffère sensiblement entre les « jeunes » et les « vieux » : « Ma socialisation militante est complètement « old school», par les livres, par les contacts humains. Les nouveaux là, ils n'ont pas de mentor, c'est internet 
leur mentor. J'ai créé libert-e ${ }^{37}$, j'ai écrit des notes pour Contrepoints.fr, j'ai fait des notes pour la Fondapol, je milite. Ce que je reproche à la génération actuelle, c'est qu'ils arrivent au libéralisme par Internet, t'arrives à leur réunion: "j'aime la liberté, pourquoi ? C'est chouette!». On a du mal à saisir les modalités de leur militantisme. Il y a plus de gens, mais moins de dimension doctrinale. »

\section{Conclusion}

Le militantisme libéral supporte une ambiguïté fondamentale : comment promouvoir par les institutions étatiques existantes la libération des individus de ces mêmes cadres réglementaires et normatifs?

En France, où l'opposition n'a juridiquement pas d'espace réservé, la contestation se réfugie dans des espaces disparates, composites, comme « la rue », les clubs et associations de la société civile. Dans ce contexte, tout effort concerté et central pour normer l'opposition libérale s'avérerait probablement illibéral. Une des solutions pour surmonter le paradoxe de l'institutionnalisation du militantisme libéral réside dans internet, technologie qui procède d'une philosophie éminemment libérale.

L'organisation réticulaire du mouvement libéral radical, qui lui vient d'un clubisme à la française doté d'un effet de résilience très fort, ne s'est pas trouvée bouleversée par l'utilisation d'internet. Le réseau se présente toujours comme un centre dynamique qui contient les forces militantes les plus vives, autour duquel gravitent des acteurs divers, plus spécialisés, et aux pratiques militantes plus dilettantes. Ainsi, il ressort de ce travail que le web a fait émerger de nouvelles formes de mobilisations militantes, de nouveaux sites au contenu très militant. Internet a également permis d'attirer un public plus jeune et bien pourvu en savoirs numériques, mais adoptant des pratiques militantes nouvelles, moins attachées à la théorie, aux grands auteurs et aux « vieux », à la vieille garde historique du mouvement.

Le champ sociologique qui traite des nouveaux mouvements sociaux demeure donc très ouvert. Après les critiques de Erik Neveu ${ }^{38}$ contre l'expression " nouveaux mouvements sociaux » elle-même, soulignant la fermeture de l'expression comme si l'on interdisait à ces mouvements sociaux qu'ils soient eux-mêmes dépassés, l'influence d'internet sur la protestation organisée n'est pas clairement établie. Peut-être la sociologie d'inspiration tourainienne, qui dérive l'état de la contestation des rapports sociaux de production et des outils techniques de communication, n'a-t-elle rien à faire du dispositif unique et gigantesque qu'est internet. Les nouveaux mouvements sociaux accusaient en effet le passage de la société industrielle à la société programmée ou post-industrielle. Tant qu'il n'est pas entendu qu'internet modifie les rapports sociaux de production et abolit les cadres traditionnels de la société programmée, une sociologie strictement tourainienne ne peut guère faire d'internet autre chose qu'un accident, au sens ontologique du terme, un trait de caractère contingent dans l'histoire des mouvements sociaux, histoire qui dérive, dans le paradigme tourainien, de l'histoire des rapports sociaux de production. 


\title{
Annexe : Détail des cinq groupes
}

\author{
site \\ https://www.contrepoints.org/ \\ http://www.ifrap.org/ \\ http://blog.turgot.org/ \\ http://h16free.com/ \\ http://www.objectifliberte.fr/ \\ http://www.institutmolinari.org/ \\ http://extremecentre.org/ \\ http://www.insolent.fr/ \\ http://www.ndf.fr/ \\ http://www.dreuz.info/ \\ http://www.bastiat.net/fr/ \\ https://leblogalupus.com/ \\ http://fr.irefeurope.org/ \\ http://www.institutcoppet.org/ \\ http://www.antagoniste.net/ \\ http://fboizard.blogspot.fr/ \\ http://www.economiematin.fr/ \\ https://olivierdemeulenaere.wordpress.com/ \\ http://www.les4verites.com/ \\ http://www.claudereichman.com/ \\ http://www.aleps.paris/ \\ http://leseconoclastes.fr/ \\ http://blog.georgeslane.fr/ \\ http://sully1.typepad.com/ \\ http://www.dumait.fr/ \\ http://www.resiliencetv.fr/ \\ http://lemennicier.bwm-mediasoft.com/ \\ http://reseauliberte.ning.com/ \\ http://www.lecercledesliberaux.com/ \\ http://laissez-faire.ch/ \\ http://les-liberes.fr/ \\ https://ecoparis.wordpress.com/ \\ http://www.tvlibertes.com/ \\ http://www.michelsanti.fr/ \\ http://eric-verhaeghe.entreprise.news/ \\ https://lilianeheldkhawam.com/
}

groupe

1

1

1

1

1

\author{
1
}

https://www.generationlibre.eu/ 3

http://economie-analyses-actualites-opinions.over-blog.com/ 3

https://jesrad.wordpress.com/ 3

http://uplib.fr/wiki/Accueil 3

http://liberalisateur.blogspot.fr/ 3

http://lefrenchlibertarien.fr/ 3

http://www.historionomie.com/ 3

https://leblogdenathaliemp.com/ 3

https://minarchiste.wordpress.com/ 3

http://www.partiliberaldemocrate.fr/ 3

http://www.libreafrique.org/ 3

http://collectifantigone.fr/ 3

http://www.dantou.fr/ 3

http://www.trop-libre.fr/ 3

http://ordrespontane.blogspot.fr 3

http://philippeherlin.blogspot.fr/ 3

http://www.ecoleliberte.fr/ 3

http://thinkliberal.org/ 3

http://studentsforliberty-france.fr/ 3

http://www.fondapol.org/ 3

http://michel-leter.blogspot.fr/ 4

http://www.temoignagefiscal.com/ 4

https://marcsuivre.wordpress.com/ 4

http://www.jbnoe.fr/ 4

http://sulfureetcontreculture.blogspot.fr/ 5

http://www.cjg.be/ 5

http://www.parti-libertarien.be/ $\quad 5$

http://www.medlib.ch/fr/ 5

http://www.pauljorion.com/blog/ 5

http://www.algarathselect.com/fr/ 5

https://yvesmontenay.fr/ 5

http://www.eradiquons.fr/ 5

http://palingenesie.com/ 5

https://www.hacklapolitique.fr/ 5

https://www.libertesociale.eu/ $\quad 5$ 


\section{$R \cdot E ́ E \cdot E \cdot E \cdot E \cdot N \cdot C \cdot E \cdot S$}

N. BAYGERT, «L'activisme numérique au regard du consumérisme politique : Pirates et Tea Partiers sous la loupe », Participations, 2014/1 $\mathrm{N}^{\circ}$ 8, p. 75-95.

J.-S. BEUSCART, E. DAGIRAL, S. PARASIE Sociologie d'Internet, Armand Colin 2016 O. BLONDEAU avec la collab. de L. ALLARD, dirs., Devenir média. L'activisme sur Internet, entre défection et expérimentation Paris, Éd. Amsterdam, 2007.

D. BOULLIER, Plates-formes de réseaux sociaux et répertoires d'action collective. Colloque "Mouvements sociaux en ligne face aux mutations socio-politiques et au processus de transition démocratique", Avril 2012, Tunis, Tunisia. Karthala, p. 37-50.

P. BOURDIEU, La Distinction. Critique sociale du jugement, Les Éditions de Minuit, 1979, $670 \mathrm{p}$.

$\mathrm{K}$. BROOKES, « Deux réseaux de promotion du néo-libéralisme entremêlés dans les années 1960 et 1970 : L'A.L.E.P.S et le groupe des Nouveaux Economistes ", Dominique Barjot, Olivier Dard et Frédéric Fogacci (dir.), Libéralisme et libéraux en Europe occidentale à l'époque contemporaine, Nouveau Monde Éditions, 2010.

J. CAMPBELL et O. PEDERSEN The National Origins of Policy Ideas: Knowledge Regimes in the United States, France, Germany, and Denmark Princeton University Press 2014.

D. CARDON, Médiactivistes (avec Fabien Granjon), Paris, Presses de Science-Po, 2010.

D. CARDON, La Démocratie internet. Promesses et limites, Paris, Seuil, 2010.
D. CARDON, « Comment s'auto-organise la production des savoirs sur Wikipédia ? ", in Larqué Lionel \& Pestre Dominique (dir.), Les sciences, ça nous regarde, Paris, Les empêcheurs de penser en rond/La Découverte, 2013, p. 266-272.

D. CARDON, G. FOUETILLOU, C. ROTH (2011), " Two Paths of Glory », Proceedings AAAI ICWSM'11 Conference Weblogs and Social Media.

S. CARÉ, Les Libertariens aux États-Unis : Sociologie d'un mouvement asocial, PUR collection Res Publica.

M. CASTELlS, La Galaxie Internet. Paris: Fayard, 2001.

A. CASILLI, Les liaisons numériques: Vers une nouvelle sociabilité?, Seuil, 2010.

S. CÉLÉRIER, Textualité numérique et mobilisation. Analyse des sites Internet d'associations françaises de lutte contre le VIH-sida. Colloque "Usages militants de la technique : technologies, medias, mobilisations », Nanterre, 12-13 mars 2009.

G. CHÂTON et S. CARÉ, « Néolibéralisme(s) et démocratie(s) », Revue de philosophie économique, vol. XVII, n ${ }^{\circ}$, septembre 2016.

F. DENORD, « Chapitre 4. Géométrie des réseaux sociaux ", in Frédéric Lebaron et al., $\mathrm{La}$ méthodologie de Pierre Bourdieu en action, Dunod « Psycho Sup », 2015, p. 59-78.

F. DENORD, Le néo-libéralisme à la française. Histoire d'une idéologie politique ( $2^{2}$ édition revue et actualisée), Marseille, Agone, 2016.

D. W. DREZNER \& H. FARRELL The Power and Politics of Blogs. Public Choice, 134, 15-30, 2008.

J., ELLUL, en particulier dans Le Système technicien Calmann-Lévy. $3^{e}$ édition Paris : Le 
Cherche-midi, 2012.

P. FLICHY, L'imaginaire d'Internet, La Découverte, Paris, 2001, 273 p.

W. GAMSON, The strategy of social protest, Paperback 1975.

R. GIBSON, S. WARD, « Renouveler le parti ?

Les stratégies de campagnes et d'organisation en ligne des partis britanniques », dans F. Greffet (dir.), Continuerlalutte.com...,

F. GRANJON, L'Internet militant. Mouvement social et usages des réseaux télématiques, collection Médias et nouvelles technologies, Rennes, Apogée, 2001.

T. JORDAN, S'engager! Les nouveaux militants, activistes, agitateurs... Éditions Autrement, collection « Frontières », 2003, 141 p.

$\mathrm{G}$. KOENIG, Le révolutionnaire, l'expert et le geek, Plon, 2015.

B. LOVELUCK, La liberté par l'information soutenue le 4 décembre 2012, EHESS.

C. MABI, A. THEVIOT « Présentation du dossier. S'engager sur Internet. Mobilisations et pratiques politiques », Politiques de communication, 2/2014, $\mathrm{N}^{\circ} 3$, p. 5-24.

J. MCCARTHY, M. ZALD, « Resource mobilizations and social movements : a partial theory » American Journal of sociology Vol. 82, Nº (May, 1977), p. 1212-1241.

R. K. MERTON, "On social structure and science” ISBN 0-226-52071-4, p. 11.

E. MOROZOV, The Net Delusion : The Dark Side of Internet Freedom, Public Affairs, 2011.

E. NEVEU, Sociologie des mouvements sociaux, La Découverte (Repères), Paris, 1996, $4^{\mathrm{e}}$ édition en 2004.

M. OFFERLÉ, Retour sur les répertoires de l'action collective, Politix, 2008/81.

M. OFFERLÉ, Sociologie des organisations pa- tronales, Paris, La Découverte, 2009, 124 p.

I. PEREIRA, « Les nouvelles technologies de l'information et de la communication et la question de la transformation de la nature des pratiques militantes à l'heure du renouveau de la contestation ». Communication donnée le 26 février 2009 au colloque Usages militants de la technique.

M. REINERT, (1986). Un logiciel à Analyse Lexicale [ALCESTE]. Les Cahiers de l'Analyse des Données, 4, 471-484.

D. RESNICK, M. MARGOLIS, Politics as usual. The Cyberspace "Revolution", Sage, 2000. F. SAWICKI et J. SIMÉANT, « Décloisonner la sociologie de l'engagement militant. Note critique sur quelques tendances récentes des travaux français », Sociologie du travail, 51 (1), 2009, p. 97-125.

S. SCHEHR, «L'alerte comme forme de déviance : les lanceurs d'alerte entre dénonciation et trahison », Déviance et Société, 2/2008 (Vol. 32), p. 149-162.

I. SOMMIER, Le renouveau des mouvements contestataires à l'heure de la mondialisation, 2003, Flammarion.

D. TARTAKOWSKY, Le pouvoir est dans la rue: crises politiques et manifestations en France, Aubier, "Collection historique », Paris, 296 p.

A. THEVIOT, «Qui milite sur Internet ? Esquisse du profil sociologique du « cyber-militant » au PS et à l'UMP », Revue française de science politique, 3/2013 (Vol.63).

A. TOURAINE, La voix et le regard, Paris, Le Seuil, 1978 [cité dans Sciences humaines, $\mathrm{n}^{\circ} 144$, déc. 2003].

F. TURNER, Aux sources de l'utopie numérique : De la contre culture à la cyberculture, C\&F Éditions, 2013. 
$\mathrm{N} \cdot \mathrm{O} \cdot \mathrm{T} \cdot \mathrm{E} \cdot \mathrm{S}$

1. Au sein des libéraux, les libertariens sont les militants qui œuvrent avant tout pour la disparition progressive de l'État au nom de l'idéal de liberté individuelle.

2. Le Mouvement des Libertariens, dont le parti émane, est lui-même créé en 2013.

3. Par souci de clarté, on définira « internet » comme une technologie, un dispositif ; et le « web » l'interface réticulaire à laquelle donne accès cette technologie.

4. B. Loveluck, La liberté par l'information thèse soutenue le 4 décembre 2012, EHESS.

5. S. Caré, Les libertariens aux États-Unis : Sociologie d'un mouvement asocial, PURcollection Res Publica.

6. Par « cyberespace » nous entendons cette projection de l'espace social via un dispositif technique empreint de contraintes techniques et de normes propres. Si le terme est bien présent dans l'ouvrage de Caré, ce texte lui préférera des expressions plus précises.

7. Patrice Flichy, L'imaginaire d'internet, La Découverte, Paris, 2001, 273 p.

8. Le terme traverse toute l'œuvre de Friedrich Hayek.

9. Les libertariens aux États-Unis : Sociologie d'un mouvement asocial, PUR, collection Res Publica, 2010.

10. Voir Pierre Mercklé et Sylvie Octobre, «La stratification sociale des pratiques numériques des adolescents », RESET 1 | 2012.

11. On parle d'aspiration de données web en français, pour traduire le nom anglais de la méthode de « scraping ».

12. Voir S. Caré, cité ci-dessus.

13. Cette expression est utilisée par F. Hayek dans sa conférence Les Intellectuels et le socialisme.
14. Voir Hayek, F. (2002). « Les intellectuels et le socialisme » Commentaire, numéro 99,(3), 673-684.

15. Voir F. Denord, « La conversion au néo-libéralisme : droite et libéralisme économique dans les années $1980 »$, Mouvements, 35-5, 2004, p. 17-23.

16. Voir : http://www.culture.gouv.fr/Actualites/ Chiffres-cles-2018-pratiques-culturelles-diversifieeset-enjeux-numeriques

17. A. Theviot, «Qui milite sur Internet ? Esquisse du profil sociologique du « cyber-militant» au PS et à l'UMP ", Revue française de science politique, 3/2013 (Vol.63).

18. R. Gibson, S. Ward, « Renouveler le parti ? Les stratégies de campagnes et d'organisation en ligne des partis britanniques », dans F. Greffet (dir.), continuerlalutte.com...

19. E. Morozov, The Net Delusion : The Dark Side of Internet Freedom, Public Affairs, 2011.

20. Voir dans S. Cadiou, S. Dechezelles, A. Roger, Passer à l'action : les mobilisations émergentes, Coll. « Logiques politiques » (2007) L'Harmattan.

21. Caroline Frau, (2012) «L'impossible passage du virtuel au réel... » in Des radios de lutte à Internet : militantismes médiatiques et numériques, Presses de la Sorbonne, Paris.

22. La méthode ici exposée est largement inspirée de D. Cardon, G. Fouetillou, C. Roth (2011), « Two Paths of Glory ", Proceedings AAAI ICWSM'11 Conference Weblogs and Social Media.

23. Que l'on peut considérer comme la frange la plus utopique du néolibéralisme.

24. Même si les contributeurs de Wikipédia et de Wikibéral forment un sous-groupe de la population relativement restreint, il n'en demeure pas moins que, sur le principe, cette technologie contributive et décentralisée plaît aux libertariens. Voir à ce sujet les travaux de Nicolas Julien sur les contributeurs de Wikipédia https://www.marsouin.org/auteur1.html, 
de même que : Cardon D. « Comment s'auto-organise la production des savoirs sur Wikipédia ? », in Lionel Larqué \& Dominique Pestre (dir.), Les sciences, ça nous regarde, Paris, Les empêcheurs de penser en rond/La Découverte, 2013, p. 266-272.

25. https://cran.r-project.org/web/packages/rvest/ rvest.pdf

26. Ce courant est celui qui critique le plus la présence de l'État. Tout pouvoir administré, centralisé et exécutif est refusé en bloc. Ainsi, cette orientation particulière du libéralisme tranche par rapport à d'autres courant, pour lesquels un État minimal demeure pertinent. Les anarcho-capitalistes optent donc pour une défense absolue de la propriété privée.

27. C'est le blog de Jean-Gilles Malliarakis, proche des néofascistes, et de RadioCourtoisie.

28. Voir Le bluff technologique, Paris, Hachette, 2012, $3^{e}$ éd.

29. Le site de Contrepoints le définit comme tel en avril 2017 : «Contrepoints est un journal en ligne qui couvre l'actualité française et internationale sous l'angle libéral depuis 2009. A. Vintray est le rédacteur en chef du journal et Benoit Gobitz son directeur de publication. [...] Le journal publie des contributions de nombreux auteurs représentatifs de l'ensemble du spectre libéral, qu'ils soient écrivains, économistes, blogueurs, etc. » https://www.contrepoints.org/la-galaxie-liberaux-org

30. Tous les prénoms des enquêtés ici sont fictifs pour anonymisation.

31. Voir l'ouvrage de F. Denord cité ci-dessus.

32. On peut considérer que c'est aussi le cas de Économie Matin, dont le site n'est que la transcription sur Internet d'un journal plus ancien. Son créateur, Jean-Baptiste Giraud a été rédacteur en chef du site libéral Atlantico.

33. Créé en 1966 par l'industriel André Arnoux avec les membres français de la société du Pèlerin et sous l'égide de l'ALEPS, l'objectif de l'association était de réhabiliter le libéralisme économique en organisant des semaines de la pensée libérale et en remettant un prix de la pensée libérale. Cf. Brookes Kevin, « Deux réseaux de promotion du néolibéralisme entremêlés dans les années 1960 et 1970 : L'ALEPS et le groupe des «nouveaux économistes », in Histoire de l'Europe Libérale. Libéraux et libéralisme en Europe, $X V I I I^{e}-X X I^{e}$ siècle, Paris, Nouveau Monde Éditions, 2016, p. 245-264.

34. On lui doit notamment un article important qui exprime bien une prénotion démentie ci-dessus : que le MEDEF n'est certainement pas l'organisation militante du mouvement libéral. Voir « Pourquoi je claque la porte du MEDEF » relayé le 20 janvier 2011 sur le site de L'Humanité.

35. Pour les imbrications entre le réseau néolibéral et le réseau conservateur, on conseillera ici l'article http://www.publicseminar.org/2018/02/neoliberalisms-populist-bastards/

36. Ce qui est patent dans le cas de l'ALEPS et des spécialistes identifiés ici qui gravitent autour.

37. Nom du site fictif pour anonymisation.

38. Cité ci-dessus. 


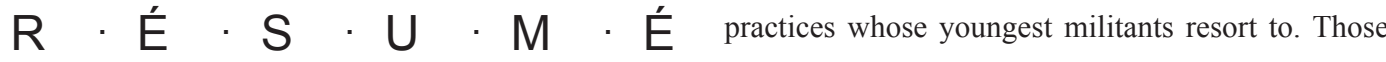 practices reshape the core of the militant network.
}

Le militantisme libertarien a adopté, en France, un mode d'organisation particulier : le « clubisme ». Cette structuration militante reposait notamment sur un réseau centralisé, et sur des têtes pensantes peu nombreuses mais très actives et légitimes aux yeux de la périphérie du réseau militant. Quel effet a eu internet, technologie dont on a immédiatement reconnu la teneur politique, sur la structure du réseau militant libertarien français, sachant qu'internet favorise les structures réticulaires décentralisées, tout à fait congruentes à la pensée libérale ? L'article conclut à l'effet de résilience très prononcé du clubisme français, et à la permanence d'un réseau militant fortement centralisé sur internet. Néanmoins, s'il ne semble pas modifier la structure de ce réseau, internet donne naissance à de nouvelles pratiques militantes dont les plus jeunes militants sont à l'initiative et qui modifient les institutions responsables du noyau du réseau militant.

\begin{abstract}
Libertarian activism has adopted a specific form of organization in France: "clubism". This structure of protest was based in particular on a centralized network, gathering few influencers that were very active and legitimate for the periphery of the protestation network. What effect had the internet, a technology whose political content had immediately been recognized, on the structure of the French libertarian activist network, knowing that the internet promotes decentralization, and that decentralized structures really fit with the liberal thought? The article concludes that French clubism is highly resilient, and that a highly centra lized internet-based activist network remains. Nevertheless, if it does not seem to change the structure of the network, the internet gives birth to new protesting
\end{abstract}


